\title{
Calibration of Electromagnetic Flow Meter
}

\author{
Kaushal Mukherjee
}

\begin{abstract}
In this paper calibration methodology of the electromagnetic flow meter is discussed. The construction and operation principle of an electromagnetic flow meter is discussed here. Installation techniques of the electromagnetic flow meter are also discussed in this paper. The typical specification of the electromagnetic flow meter is listed in this paper. All possible causes for wrong reading and possible correction are also discussed in this paper. Complete calibration process flow is represented here.
\end{abstract}

\section{Keywords}

Calibration of flow meter;

LCD flow meter;

LED flow meter;

Electromagnetic flow meter;

Magnetic flow meter;

\section{INTRODUCTION}

Flow meter means the meter, which is used to measure the rate of flow of any conductor flowing through any pipe [1]. Through which whole the conducting liquid flows is known as the flow tube and base on the pipe direction either vertical or horizontal [2]. Various kinds of flow meter are available in the market [3]; Electromagnetic flow meter is one of them, according to price, accuracy and availability [4]. Electromagnetic flow meter is also known as MICROMAG [5]. Measurement of the result is independent upon the temperature, velocity, viscosity, pressure, hard resistance until the minimum conductivity of the media remains at $5 \mu \mathrm{S} / \mathrm{cm}$. Various types of materials are used for the construction of lining and electrode to cover the major amount of corrosive liquids.

Magnetic flow meter is also known as mag flow meter or simply mag, generally used to measure wastewater application [6]. It is also used for other volumetric measure, but not suitable for distilled water and hydrocarbons [7]. Magnetic flow meter's has life span and very low maintenance cost [8]. These flow meters simply works based on Faraday's Low of Electromagnetic Induction [9]. According to this principle when a conductive medium passes through a magnetic field (B), a voltage (E) is generated. The generated voltage $\mathrm{E}$ is proportional to the velocity $v$ of the medium, length of the conductor and the density of the magnetic field. Strong electromagnet is generated by applying current to the wire coils which is mounted outside of the pipe [10]. The pipe acts as conductor. The induced voltage is proportional to the average flow velocity. The induced voltage is detected by sensing electrode which is mounted in the meter and transmit to the microcontroller for mapping to equivalent volumetric flow measurement based on pipe dimension and display in LCD [11]. In section 2, different type of electromagnetic flow meter is discussed. In section 3 , the general specifications of the electromagnetic water flow meter is represented for application. Calibration process of LCD and LED type electromagnetic flow meter is discussed in section 4 and 5 respectively.

\section{ELECTROMAGNETIC FLOW METER TYPES}

Electromagnetic flow meter is of two types-

Integral type in this type of flow meter both the PCB and display remains integrated and remote type in this type of flow meter remote and display remains in different places. Display of the flow meter can be of two types, one is LED type and another one is of LCD type. General constriction of the flow meter is shown in the Fig. 1.

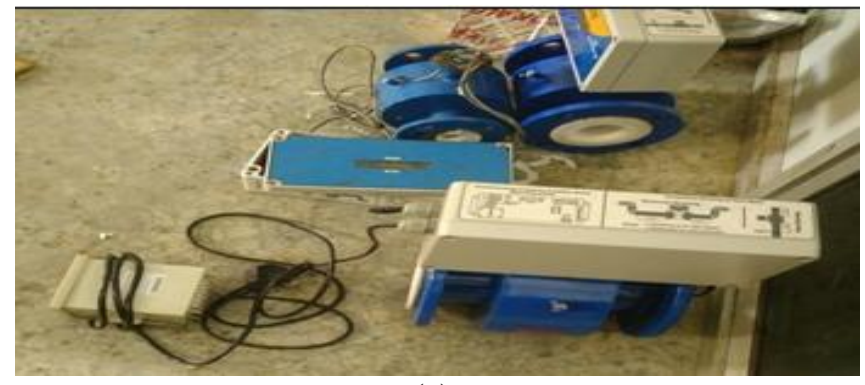

(a)

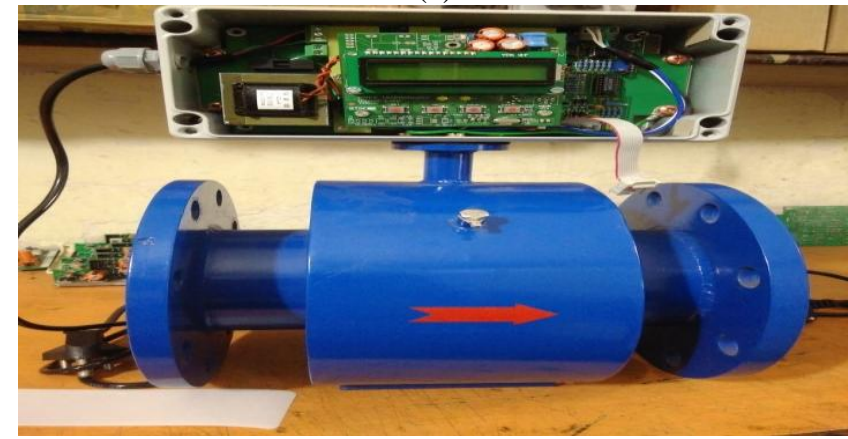

(b)

Fig 1. Electromagnetic flow meter (a) different parts of the flow meter (b) assemble view.

Electromagnetic water flow meter can be mounted in two ways:

1. Horizontally(recommended) and

2. Vertically (not recommended)

In case of vertical or horizontal installation of the flow meter, the pipe must have been horizontal for at least 10D 
before the meter and 5D after the meter otherwise the flow of media through the pipe will not be in full flow which in result provides wrong reading.

\section{SPECIFICATION OF THE ElECTROMAGNETIC FLOW METER}

The general specifications of the electromagnetic water flow meter:

1. Meter size- DN 10 to DN 1000(diameter of nominal bore)

2. Media pressure- up to DN 80-:PN 40(nominal pressure) From DN 100-DN 200: PN 16 From DN 250-DN350: PN 10

3. Media temperature:

PFA liner:0-200 ${ }^{\circ} \mathrm{C} \max$

i. PTFE liner: $0-150^{\circ} \mathrm{Cmax}$

ii. Rubber liner:0-90 $9{ }^{\circ} \mathrm{C} \max$

4. Materials-

a. Pipe: SS304(non-magnetic)

b. Electrode: SS 316/Hastelloy $\mathrm{C} / \mathrm{Pt} / \mathrm{Ta} / \mathrm{Ti}$

c. Flange: SS 316/SS 316L/ SS 304,Carbon steel

d. Liner: PFA(paraflouroaloxy)/ PTFE(teflon)/ Neoprene/ Polyurethane/ hard rubber/ soft rubber

e. Coil housing: Carbon Steel, P.U painted/SS

5. Ambient temperature range-: $0-50^{\circ} \mathrm{C}$

6. Flange standard: ANSI/BS/SMS/DIN/Tri-clamp

7. Power supply to field coils: pulsed DC

8. Type of electromagnetic flow meter:
a. Integral type

b. Remote type

9. Minimum media conductivity: $5 \mu \mathrm{S}$ (Siemens) $/ \mathrm{cm}$

10. Signal output: 4-20mADC in max $600 \mathrm{ohms}$

11. Frequency output: $0-10 \mathrm{kHz}$ prop. to $100 \%$ flow rate

12. Coil excitation frequency: selectable DIP switch
a. $25 \mathrm{~Hz}$
b. $12.5 \mathrm{~Hz}$
c. $6.25 \mathrm{~Hz}$
d. $3.125 \mathrm{~Hz}$

13. Display : in case of LED display
a. Display 1 is of 9 digit, shows the total flow

b. Display 2 is of 4 digit, shows the flow rate

14. Flow velocity range: $0.3 \mathrm{~m} / \mathrm{s}$ to $12 \mathrm{~m} / \mathrm{s}$

15. Accuracy:

a. $\pm 0.5 \%$ of meter reading between $10 \%$ to $100 \%$ of calibrated range

b. $\pm 0.75 \%$ of meter reading for flow rate between $10 \%$ to $5 \%$

16. Reference condition: power supply nominal $27^{\circ} \mathrm{C} \pm 2^{\circ} \mathrm{C}$
17. Repeatability : $\pm 0.2 \%$ of reading

18. Temperature drift: $\pm 0.015 \%$ per ${ }^{\circ} \mathrm{C} \max$

19. Humidity : $90 \%$ R.F max non-condensing

20. Material of housing: AL. die cast

21. Power supply:
a. $230 \mathrm{~V} \mathrm{AC}, 50 \mathrm{~Hz} / 24 \mathrm{~V} \mathrm{DC}$
b. $110 \mathrm{~V} \mathrm{AC}, 50 \mathrm{~Hz} / 24 \mathrm{~V} \mathrm{DC}$

22. Damping : adjustable from 5 to $30 \mathrm{~s}$

23. Cable entries:
a. 2 no of entries for integral type
b. 4 no of entries for remote type
$1 / 2^{\prime \prime} \mathrm{NPT} / 1 / 2{ }^{\prime \prime} \mathrm{BSP} / \mathrm{PG} 11$

24. Ingress protection: IP-65.

\section{CALIBRATION PROCESS OF LCD TYPE ELECTROMAGNETIC FLOW METER}

Available keys of LCD type electromagnetic water flow meter are which are available to the user are SET, UP, DOWN and ENTER.

After setting the meter with the pipeline, firstly you have to make the zero, that means the total flow and the flow rate must be 0 . The flow meter can show OPEN, that means the power supply connected with the PCB board is faulty, or the cable which connects the PCB and the power supply is faulty. To overcome these problems change the particular faulty device or the cable. The flow meter can show, OVER, that means the meter reading exceeds the higher range fixed in the meter. To overcome this problem, set the upper range as the standard higher range. The flow rate may not be zero, this is because the output current is not between 4-20 mA. To solve this problem, set the output current value to $0.37 \mathrm{~mA}$. when the value of output current reaches $0.37 \mathrm{~mA}$ the flow rate will be consequently reaches 0 value. The minimum output current value is set to $0.37 \mathrm{~mA}$, but not to $4 \mathrm{~mA}$ as because of many industry, the value of current of $0.1 \mathrm{~mA}$ can damage the flow meter, so for the safety purpose, the value of output current is been set to the $0.37 \mathrm{~mA}$. Secondly make the lower range as 0000 and upper range as per standard or as per the customer requirement, with the help of UP and DOWN key, shift of digit is done with the help SET key. After changing the upper range, shift the digit to the first digit. Suppose upper range is 50, set the range as 50.00 and when the first digit means 5 will blink then only you can go to the next step. Then change the unit as LPM, LPH, LPS or $\mathrm{M}^{3} / \mathrm{hr}$, as per the requirement of the customer. After changing the unit, the remaining data's will stay's same as it set from previous.

Next step is to RESET the meter, the process of reset the meter is, press the SET key for 2second, then the display will ask for a password, enter the UP key for 3 times as password, then after 2 second press the UP key again, now 
the display will show, press SET to cancel the RESET process or press ENT to reset. To reset press ENT or to cancel press SET. After resetting the meter, again check the output current value, if it is $0.37 \mathrm{~mA}$ then $\mathrm{OK}$, no problem; proceed to the final step otherwise set the output current value as $0.37 \mathrm{~mA}$ by increasing or decreasing the resistance of the ZERO pot.

After setting the output current, RESET the meter again and after then, switch ON the pump, to flow the conductor through the meter. To calibrate the meter change the value of the resistance which is present at the pot, named as range change pot. Formula for the calculation of the resistance required for calibration process is:

Total flow passing through the meter/flow rate=time

So, flow rate=total flow showing by the weight machine/time

If the result is same as the flow meter reading of flow rate, that means the meter is calibrated otherwise, we have to make sense that the meter is not still calibrated, so subtract the value of actual flow rate from the meter's flow rate, and change the resistance $f$ same amount by varying the range changing pot. After the calibration process, reset the meter again for final test. This is all about the calibration process of the LCD type electromagnetic water flow meter.

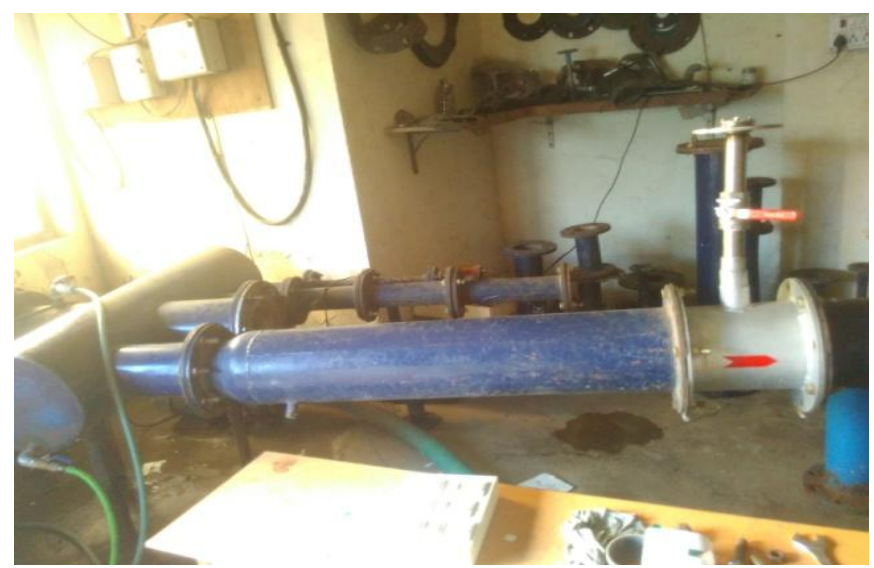

(a)

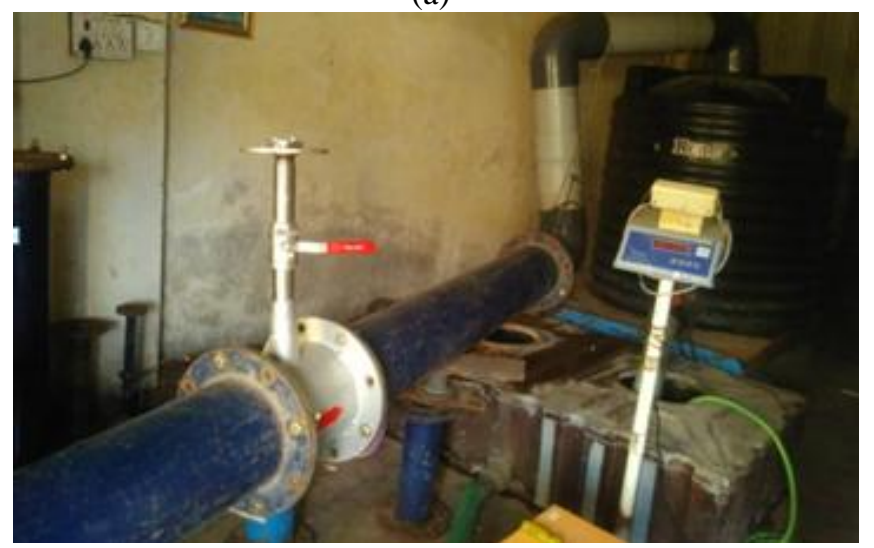

(b)
Fig 2. Mounting process of water flow meter.

\section{CALIBRATION PROCESS OF LED TYPE ELECTROMAGNETIC WATER FLOW METER}

The display of the LED type electromagnetic flow meter consists of four switches/buttons, those are

Set/Enter, Shift, Increment/Decrement and Reset from left to right side respectively.

To install an electromagnetic flow meter, you first have to change the lower value, upper value, unit and decimal point (which is abbreviated as $\mathrm{dP}$ ).

Lower value will remains at 0000 in maximum cases, but if customer has any requirement then the lower value can be set at negative also.

Upper value is set as per the customer requirement.

Unit Is been set as LMP, LPS, LPH, $\mathrm{M}^{3} / \mathrm{Hr}$ or gallons/minute

$\mathbf{d P}$ can be set as one decimal or two decimal point or three decimal point.

In the calibration process you have to fix two things, those ares-

1. Fix the output value as 37 , which is standard, by changing the zero pot and span pot

2. Calibrate the meter according to the weight of the total flow flowing through the pipe in a minute, second or hour.

Accuracy of a electromagnetic flow meter is $\pm 5 \%$.

The main operation principle for this method is known as the "Faraday's Law', which states that, when a conductor moves in a magnetic field a voltage is induced in it which is proportional to the rate of flow of the conductor or the velocity of the conductor [12].

Mathematically,

$\mathrm{E}=\mathrm{K} . \mathrm{B} . \mathrm{V} . \mathrm{D}$

Where,

$\mathrm{K}=$ proportionality constant

$\mathrm{B}=$ magnetic flux density

$\mathrm{V}=$ mean velocity of the media

$\mathrm{E}=$ induced voltage

$\mathrm{D}=$ distance between the electrodes

For a given size of flow tube and compatible amplifier flux density $\mathrm{B}$, is constant, the distance between the electrodes is constant [13]. Hence, the induced voltage is proportional to the velocity of the flowing media. So, the unit can be calibrated in terms of volumetric flow rate by knowing the cross sectional area of the tube.

Flow tube- flow tube is the tube, through which the conductor flows in the meter.

The meter always measures volume rate hence the volume of any suspended matter in the liquid also included in the 
measurement. The measurement result may be affected by incomplete full run. Zero check on the installation can be performed by stopping flow.

\section{Conclusion}

Magnetic flow meter can be used to measure dirty, corrosive and abrasive liquid flow but it does not suitable for nonconductive liquid. Magnetic flow meters required 35 diameters of upstream straight run. Proper calibration is required if the result is same with the reference flow meter as the flow meter reading of flow rate, that means the meter is calibrated otherwise, the meter is not still calibrated, so subtract the value of actual flow rate from the meter's flow rate, and change the resistance $f$ same amount by varying the range changing pot. There may be a chances of sucked into the pipeline in vacuum which may damaging the magnetic flow meters.

\section{REFERENCES}

[1] Hess, A. E. (1986). Identifying hydraulically conductive fractures with a slow-velocity borehole flowmeter. Canadian Geotechnical Journal, 23(1), 6978.

[2] He, Y., Jin, Y., Chen, H., Ding, Y., Cang, D., \& Lu, H. (2007). Heat transfer and flow behaviour of aqueous suspensions of $\mathrm{TiO} 2$ nanoparticles (nanofluids) flowing upward through a vertical pipe. International Journal of Heat and Mass Transfer, 50(11), 22.

[3] Falcone, G., Hewitt, G. F., Alimonti, C., \& Harrison, B. (2001, January). Multiphase flow metering: current trends and future developments. In SPE annual technical conference and exhibition. Society of Petroleum Engineers.

[4] Hristoforou, E., Avaritsiotis, I. N., \& Chiriac, H. (1997). New flowmeters based on amorphous magnetic wires. Sensors and Actuators A: Physical, 59(1-3), 9496.

[5] Hristoforou, E., Avaritsiotis, I. N., \& Chiriac, H. (1997). New flowmeters based on amorphous magnetic wires. Sensors and Actuators A: Physical, 59(1-3), 9496.

[6] Pereira, M. (2009). Flow meters: part 1. IEEE Instrumentation \& Measurement Magazine, 12(1), 1826.

[7] Busch, K. W., Busch, M. A., Parker, D. H., Darling, R. E., \& McAtee Jr, J. L. (1986). Studies of a water treatment device that uses magnetic fields. Corrosion, 42(4), 211-221.

[8] Michalski, A., \& Jakubowski, J. (2011). An energy- saving algorithm for electromagnetic flow measurement in open channels [Instrumentationnotes. IEEE Instrumentation \& Measurement Magazine, 14(6).

[9] Blakeley, C. P., \& Thomas, T. K. (1973). Instrumentation for water pollution monitoring. Environmental science \& technology, 7(11), 10061010 .

[10] Watral, Z., Jakubowski, J., \& Michalski, A. (2015). Electromagnetic flow meters for open channels: current state and development prospects. Flow measurement and Instrumentation, 42, 16-25.

[11] Yao, Jun, WeiGuo Wang, and Ji Shi. Study on electromagnetic flowmeter for partially filled flow measurement. In Control and Decision Conference (CCDC), 2011 Chinese, pp. 3568-3573. IEEE, 2011.

[12] Scott, M. J. (1995). Process Sensing and Control. Sensors Set: A Comprehensive Survey, 413-430.

[13] Coursolle, Thomas P., and David L. Wehrs. Electrode leakage diagnostics in a magnetic flow meter. U.S. Patent 6,611,775, issued August 26, 2003.

\section{Author Details \\ Kaushal Mukherjee \\ Dept. of ECE, \\ Bankura Unnayani Institute of Engineering, Bankura, 722146, India \\ mukherjeek95@gmail.com}

